\title{
eHealth and mHealth Interventions for Ethnic Minority and Historically Underserved Populations in Developed Countries: an Umbrella Review
}

\author{
Maria Armaou $^{1}$ (D) Evangelia Araviaki ${ }^{2}$. Laura Musikanski ${ }^{3}$
}

Received: 7 September 2019 / Accepted: 17 December 2019 / Published online: 30 December 2019

(C) The Author(s) 2019

\begin{abstract}
Health disparities among historically disadvantaged populations can undermine the effectiveness of eHealth and mHealth interventions and limit their acceptability among diverse community members. The twin aims of this umbrella review of systematic reviews (SRs) are to summarise the evidence on the characteristics and effectiveness of eHealth and mHealth interventions among underserved populations in developed counties and provide recommendations to community organizers, policy makers and researchers. Comprehensive searches were conducted in bibliographic databases, Google Scholar and references lists for SRs published in English between 2000 and 2019. SRs were selected following a protocol registered with PROSPERO. Two independent reviewers were involved in the selection appraisal process, quality assessment and data extraction process. Six SRs met the inclusion criteria for this umbrella review. The six SRs concerned interventions delivered by computer programmes, cell-phones or other electronic devices. The studies in the SRs reported improvements to physiological wellbeing, health knowledge and self-management, as well as improvements in psychosocial outcomes. This umbrella review concludes with recommendations for community organizers, policy makers and researches for the formation of guidelines, inclusion of target community members in the development of eHealth interventions and directions for future research.
\end{abstract}

Keywords eHealth $\cdot \mathrm{mHealth} \cdot$ Systematic reviews $\cdot$ Health disparities $\cdot$ Underserved communities

Electronic supplementary material The online version of this article (https://doi.org/10.1007/s42413-01900055-5) contains supplementary material, which is available to authorized users.

Maria Armaou

vavel7@yahoo.gr

Laura Musikanski

laura@happycounts.org

Extended author information available on the last page of the article 


\section{Introduction}

The twin aims of this umbrella review is to assess the state of the evidence for the effectiveness of eHealth and mHealth interventions for minority and historically underserved populations in developed counties and provide recommendations for community organizers, policy makers and researchers. A precise definition of eHealth and mHealth can be challenging due to the continuing advancements in technology and the breadth of technological applications. For the purposes of this umbrella review, eHealth is defined as health services delivered on the internet or other technological means (Eysenbach 2001) and mHealth is a subset of eHealth (World Health Organization 2012a, b) health services delivered on mobile devices (i.e. mobile phones, personal digital assistants) (Germanakos et al. 2005).

Umbrella reviews constitute a synthesis of the evidence derived from multiple systematic reviews (SRs). An umbrella review assesses the quality of SRs, explains inconsistencies and highlights areas for improvement for future research (Aromataris et al. 2015, 2017). Umbrella reviews can be useful for community organizers and policy makers, as well as for the development of guidelines, as they provide a review of a large amount of information in an easily accessible condensed format (Ioannidis 2009).

\section{Community Well-Being and the Role of eHealth and mHealth Interventions for Ethnic Minority and Historically Underserved Populations in Developed Countries}

Health inequalities are defined as differences in the health status or the distribution of health determinants between different population groups (World Health Organization 2012a, b). There are with complex causes and wide-spread effects to community wellbeing due to economic inequality, political decisions, the wider socio-economic environment, societal values, and marginalisation (NHS Health Scotland 2016; Phelan et al. 2010; Popay et al. 2015; Bharmal et al. 2015). Moreover, health inequalities influence educational and work opportunities, access to health services and ultimately have negative effects on well-being and healthy life expectancy (NHS Health Scotland 2016; Phelan et al. 2010; Popay et al. 2015; Bharmal et al. 2015).

There are links between socioeconomic inequity, health and community well-being (Srinivasan et al. 2003). The burdens of illness are greater among minorities and lowincome communities that live in neighbourhoods without adequate healthy food options, opportunities for outdoor activities, access to quality housing, high crime rates and social isolation (Hoffman 2019; Srinivasan et al. 2003; Popkin et al. 2005; Kohlhuber et al. 2006). Stiglitz et al. (2009) called for the prioritization of well-being over economic growth in part because of inequality and the burden on the well-being of those suffering from inequality. In the first World Happiness Report, physical and mental health were cited as "key determinants" (Layard et al. 2012, p. 59) of wellbeing. In the seventh World Happiness Report, Helliwell et al. (2019) synopsize prior World Happiness Reports, stating that "inequalities in the distribution of health have effects on life satisfaction above and beyond those flowing through their effects on income" (p. 18). In the same report, Bellet and Frijters (2019) identify problems associated with health care applications (apps) including (1) privacy and consent of the user, (2) sale by the app provider and use of data by insurance companies and (3) 
the concern that "Big Data might increase the importance of sheer computing power and data storage capacity, both likely to favour capital and thus increase inequality whilst reducing median wages" (p. 116). However, Bellet and Frijters (2019) do not mention the issues of equal and equitable delivery to healthcare apps and inequality.

There is evidence that eHealth and mHealth services yield socio-economic benefits (Koutras et al. 2015; Price Waterhouse Coopers 2013). Ensuring equal and equitable delivery of health care is often seen as a community-based activity in developed and developing nations (Adashi et al. 2010; Dahn et al. 2015). However, social health inequalities appear to be increasing in developed countries in the current era of rapid innovative technological development (Mackenbach 2012).

Freimuth and Quinn (2004) recognize that in order to design interventions that are effective, cultural sensitivity is needed. Collaboration with communities experiencing health disparities is also necessary for the effectiveness of eHealth interventions (Freimuth and Quinn 2004). Kontos et al. (2014) state that the examination of the effectiveness of eHealth and mHealth interventions, including interventions for acute and chronic conditions to preventative care across different racial and social groups, is important. Studies suggest that certain interventions (for example HIT DSME interventions for diabetics in the Heitkemper et al. 2017 study) show small but significant improvement in underserved populations.

Despite the growing popularity of eHealth and mHealth interventions, there is limited evidence about their scalability and long-term impact on health outcomes due to the low quality of the evidence, particularly from umbrella reviews and overviews of previous SRs (Marcolino et al. 2018). Schueller et al. (2019) state "much work still needs to be done" (p. 252) to develop digital mental health interventions for marginalized and underserved populations, in part because of SR findings that a number of pilot studies have not "follow[ed]-up with an implementation component" (p. 251). It is important to conduct high quality SRs on minority populations as they face discrepancies in accessing mental health services and, hence, poor treatment outcomes is not uncommon (Masood et al. 2019). SRs conducted in the context of developing countries suggest that the impact of mHealth should be assessed based on the extent to which they influence behaviour leading to an improvement of public health services and the degree to which they take into consideration local needs (Aranda-Jan et al. 2014; Chigona et al. 2012). Arak and Wójcik (2017) state that the differences between EU countries need to be addressed when developing and implementing eHealth and mHealth interventions. They recommend that eHealth and mHealth implementation takes into consideration the characteristics of regional public and private health systems; the production of high quality standards equal to those that apply to other healthcare products; the use of the experience of previous public-private partnerships; an increase in collaboration with eHealth research and development teams; and the promotion of eHealth and mHealth solutions across age groups in both rural and urban areas to increase digital health literacy within communities and their healthcare professionals.

\section{Objectives of this Umbrella Review}

The overall aim of this umbrella review is to provide evidence-based recommendations for community organizers, policy makers and researchers seeking to mend healthcare 
inequality for communities through the delivery of eHealth and mHealth interventions with the intent of contributing to an understanding of which evidence-based strategies contribute to mending social health inequalities and also contribute to the effectiveness of interventions. The means used to achieve this aim is the rigorous synthesizing of findings of SRs on the effectiveness of eHealth and mHealth interventions for ethnic minority or historically underserved populations in developed countries and an evaluation of the characteristics and limitations that exist in eHealth and mHealth interventions targeting specific populations within specific contexts.

\section{Methodology}

A protocol was developed and registered with PROSPERO, the international prospective register of systematic reviews (registration number: CRD42019129123). No major violations of the protocol were identified in the research procedures. However, the title of the registered protocol includes a focus on economically underserved populations, whereas this umbrella review focuses on ethnic minority and historically underserved populations. A PRISMA statement is provided in Appendix 1.

SRs were included or excluded for this umbrella review based on three criterions. They are listed below as:

1. SRs must have explicitly used a systematic process to the literature search and study selection.

2. SRs must have identified empirical research evidence related to the subject

3. For SRs published between January 2000 and March 2019, SRs must have met the population, interventions, comparison, and outcomes (PICO) derived inclusion criteria.

\section{Population}

SRs were considered eligible for this umbrella review if they explicitly reported that their target populations belonged to an ethnic minority or a historically disadvantaged or underserved population within communities. Ethnic minorities differ within different countries. It has been argued that the use of the term minority can perpetuate inequalities (Larson 1999; Montague and Perchonok 2012). For this reason, in this umbrella review, the term historically underserved refers to individuals that have limited or no adequate access to healthcare services due to a range of characteristics (e.g. race, ethnicity, gender, socioeconomic status, or geographic location). By the authors' judgement, the term historically was used as it better reflects the target populations of this umbrella review and as the inclusion/exclusion criteria is not solely based on socio-economic status. SRs included in this umbrella review reported about studies that focused on such groups. SRs were excluded if they focused on adolescents (under 18 years of age) or solely on healthcare service outcomes from the perspective of healthcare providers (e.g. physicians or general practitioners, healthcare workers, nurses, etc).

The Central Intelligence Agency (CIA) World Factbook appendix was used to determine which countries are developed countries. The developed countries are: 
Australia, Austria, Belgium, Canada, Cyprus, Czech Republic, Denmark, Estonia, Finland, France, Germany, Greece, Iceland, Ireland, Israel, Italy, Japan, Luxembourg, Netherlands, New Zealand, Norway, Portugal, Slovenia, South Korea, Spain, Sweden, Singapore, Switzerland, Turkey, United Kingdom, and United States (CIA 2007). Developed countries were defined, per the authors' judgement, as countries with a high standard of living, high level of economic security and growth and advanced technological infrastructure.

\section{Interventions}

SRs reporting patient-centred interventions using an eHealth modality delivered via computer, web, text, mobile phone, applications, email or related technologies were included. SRs that solely reported mass-media interventions, interventions delivered only via non-interactive technology (e.g. CDs, DVDs) or interventions solely available to health care providers, health care clinics, hospitals or other health care professional institutions or representatives were excluded.

\section{Outcomes}

SRs that primarily reported measurable health or mental health outcomes assessed at any point after enrolment in a study were included. If SRs did not report measurable health or mental health outcomes, they were excluded. For this reason, SRs reporting on secondary outcomes were only included if they reported on measurable health or mental health outcomes. Secondary measurable outcomes included (1) assessment of health behaviour changes, (2) intentions to change personal habits, (3) adherence to a treatment/intervention, (4) improvements to quality of life, (5) digital literacy and (6) acceptability of the interventions.

\section{Types of Studies}

SRs that reported experimental (e.g. randomised controlled trials), quasi-experimental studies (e.g. non-randomised trials) or mixed-methods studies (e.g. combinations of randomised and non-randomised trials and feasibility studies) were included. SRs that included studies reporting a mix of web-based, computer-based and technology-based (e.g. computer-based, mobile-based, or other non-internet means) interventions were included. SRs of cross-sectional studies (e.g. observational studies analysing questionnaire data at a specific point in time), and qualitative reviews were excluded. Grey literature (e.g. studies in process, conference presentations, book chapters, or nonacademic literature) were not included. SRs that reported studies delivered via mass media were not included.

\section{Search Strategy}

An initial search was conducted in October 2018 and updated in March 2019. A comprehensive search strategy was developed for Embase, Medline, Cumulative Index to Nursing and Allied Health Literature (CINAHL) and the Cochrane Library (see Appendix 2: full search strategy). CINAHL results were excluded from analysis as an 
initial enquiry showed only a small number of duplicate records from Embase and Medline. Searches were supplemented by hand-searching reference lists of relevant SRs and searches in Google Scholar using text terms combining multiple keywords. SRs identified through those supplemental searches were only considered for inclusion if they were published in peer-reviewed journals.

The foci of the search were (1) eHealth/telemedicine, (2) minority/underserved populations, and (3) systematic reviews/syntheses. A list of keywords was created based on the current literature about the application of internet-related technologies in health research. The search strings (i.e. combinations of words and characters) that were generated entailed widespread terms associated with the field, along with combinations of keywords and specialised queries. They were minorit* OR underserved [eHealth OR eHealth OR telemedicine OR tele-health OR information technology] and systematic ( $\mathrm{sb}$ ). Both the terms of eHealth and telemedicine were employed, as in many databases the relevant Medical Subject Heading (MeSH) descriptor was the term telemedicine, but not all authors of relevant SRs used keywords to list their SR in the same manner. For this reason, additional search strings were constructed using targeting specific keywords (see Appendix 2). Searches were limited to SRs published in the English language between 2000 and 2019 in peer-reviewed academic journals.

\section{Screening Process, Data Extraction and Synthesis}

The screening of the full-text of studies and the data extraction was done independently by two of the authors, Maria Armaou (MA) and Evangelia Araviaki (EA). MA had previous knowledge of SRs and a keen interest in digital health. MA developed the search strategy and conducted the search process. EA came into the process at the screening stage after receiving training from MA. MA and EA conducted the final stage of the screening as well as the data extraction on a mutually agreed upon final list of studies that met the inclusion criteria. Disagreements were resolved through consensusbased decisions for excluding SRs based on the inclusion criteria. The interrater reliability was $\mathrm{k}=0.81$ (Table 1 ).

Data extraction for the SRs that met the inclusion criteria was performed independently by both MA and EA using the Joanna Briggs Institute (JBI) data extraction form for SRs and research synthesis (Munn et al. 2014). The JBI data extraction form is a standardised tool that allows the extraction of all relevant information with regard to the characteristics of the included SRs and their results (Munn et al. 2014). Extracted

Table 1 Reviewer interrater reliability

\begin{tabular}{lll}
\hline & $\mathrm{N}$ & \\
\cline { 2 - 3 } Categories & Include & Exclude \\
\hline Reviewer A: & 8 & 18 \\
Reviewer B: & 7 & 19 \\
Joint coding (after discussion) & 6 & 20 \\
k Statistic & 0.81 & \\
\hline
\end{tabular}


information for each SR included the following: (1) basic study characteristics (i.e. author and year, objectives, participants, context, description of interventions, description of interventions or phenomena of interest), (2) search details (i.e. sources searched, range or years of included studies or detailed description of the included primary studies in terms of number or type of studies), (3) country of origin of studies, (4) appraisal details (i.e. appraisal instruments used or appraisal rating), and (5) analysis (i.e. method of analysis, outcome assessed, findings, significance/direction, heterogeneity or comments). Data for the populations, interventions and outcomes were extracted from the SRs and tabulated. The extraction forms were entered into an Excel spreadsheet. Inconsistencies in extraction notes were discussed and resolved through email communication and face-to-face meetings where each of the reviewers checked each other's notes.

Popay et al. 2006 guidance on the construction of narrative synthesis was followed. The guidance included the formation of textual descriptions of the studies through a tabulation process that organised studies into logical categories as well as critical reflection across the categories. MA performed a narrative synthesis on the Excel spreadsheet.

As only one SR included a meta-analysis, it was not possible to use the Grading of Recommendations Assessment, Development, and Evaluation (GRADE) tool to assess the certainty in evidence for any pooled outcomes.

\section{Quality Assessment}

The assessment of the methodological quality of the SRs was performed using A MeaSurement Tool to Assess Systematic Reviews (AMSTAR 2). AMSTAR 2 is a critical appraisal tool for SRs randomised and non-randomised studies of healthcare studies (Shea et al. 2017). It has 16 items describing its Quality Assessment Criteria (QAC) (Table 2) and allows an assessment for each SR in critical and non-critical domains. Critical domains are assessed by the following QACs: QAC2, QAC 4, QAC 7, QAC 9, QAC 11, QAC 13, QAC 15. A strength of the AMSTAR 2 tool is that it assesses the validity of a SR by addressing its critical and non-critical weaknesses (Shea et al. 2017). Reviewers can then determine the degree to which there should be high, moderate, low and critically low confidence in SRs findings (Shea et al. 2017).

AMSTAR 2 was used to evaluate the quality of the SRs that included experimental (i.e. randomized control trials [RCTs]), quasi-experimental (i.e. non RCTs) or mixed methods studies. The quality of each SR was assessed by MA and EA independently. When any discrepancies were spotted, MA and EA reached consensus through discussion.

\section{Results}

\section{Review Inclusion/Exclusion}

The literature search identified 1662 titles. 1642 were from bibliometric databases and 20 were from searches in Google scholar and hand searches in the references of other SRs (Fig. 1). After removing the duplicates $(N=184)$, the titles and abstracts of 1478 
Table 2 AMSTAR 2 items

Quality assessment criteria

QAC1 PICO components included in the review research question and inclusion criteria

QAC2 Explicit statement included that review methods were established prior to conduct and significant deviations justified

QAC3 Selection of included study designs explained

QAC4 Comprehensive search strategy used

QAC5 Study selection performed in duplicate

QAC6 Data extraction performed in duplicate

QAC7 List of excluded studies with justification provided

QAC8 Included studies described in adequate detail

QAC9 Satisfactory technique used for assessing risk of bias in included studies

QAC10 Sources of funding for included studies reported

QAC11 Appropriate methods for statistical combination of results used if meta-analysis performed

QAC12 Potential impact of risk of bias of individual studies assessed if meta-analysis performed

QAC13 Risk of bias of individual studies accounted for in discussion of the review results

QAC14 Any heterogeneity observed in the review results was explained and discussed

QAC15 Publication bias investigated and discussed if meta-analysis performed

QAC16 Authors reported any potential sources of conflict of interest

records were screened by MA. EA independently reviewed half of the papers due to workload volume and time constraints. Following this process 1452 papers were excluded for not meeting the inclusion criteria. Full text SRs assessing the eligibility of the remaining 26 SRs was undertaken by MA and EA independently. Through a consensus-based discussion, 20 records were excluded for not meeting at least two of the eligibility criteria (i.e. populations, type of interventions, type of outcomes, types of studies). Six SRs (Table 3) progressed to the quality appraisal stage (see Appendix 3 for full list of excluded studies).

\section{Methodological Quality Assessment}

According to the AMSTAR 2 tool, five SRs were classified as critically low $(n=5)$ and one as low $(\mathrm{n}=1)$ (Table 4). The QAC (See Table 2) that was not met by any of the SRs was QAC 7. All six SRs met QAC3, QC4, QC8 and QAC16. Four SRs (SR1, SR2, SR4, SR5) met QAC5 and three SRs (SR1, SR2, SR5) met QAC2 and QAC6. Three SRs (SR1, SR2, SR6) met QAC9; two SRs (SR1, SR6) met QAC 10 and QAC 14; and three SRs (SR2, SR5, SR6) met QAC13. Meta-analysis was not performed in most of the studies, hence QAC11, QAC12 and QAC15 were coded as mostly not applicable.

\section{Population and Study Characteristics of Included Reviews}

The six SRs included reviews of experimental, quasi-experimental designs, and mixed methods research designs. The six SRs included RCTs, partially randomised studies, uncontrolled trials, feasibility trials/ pilot RCTs and mixed methods research designs. 

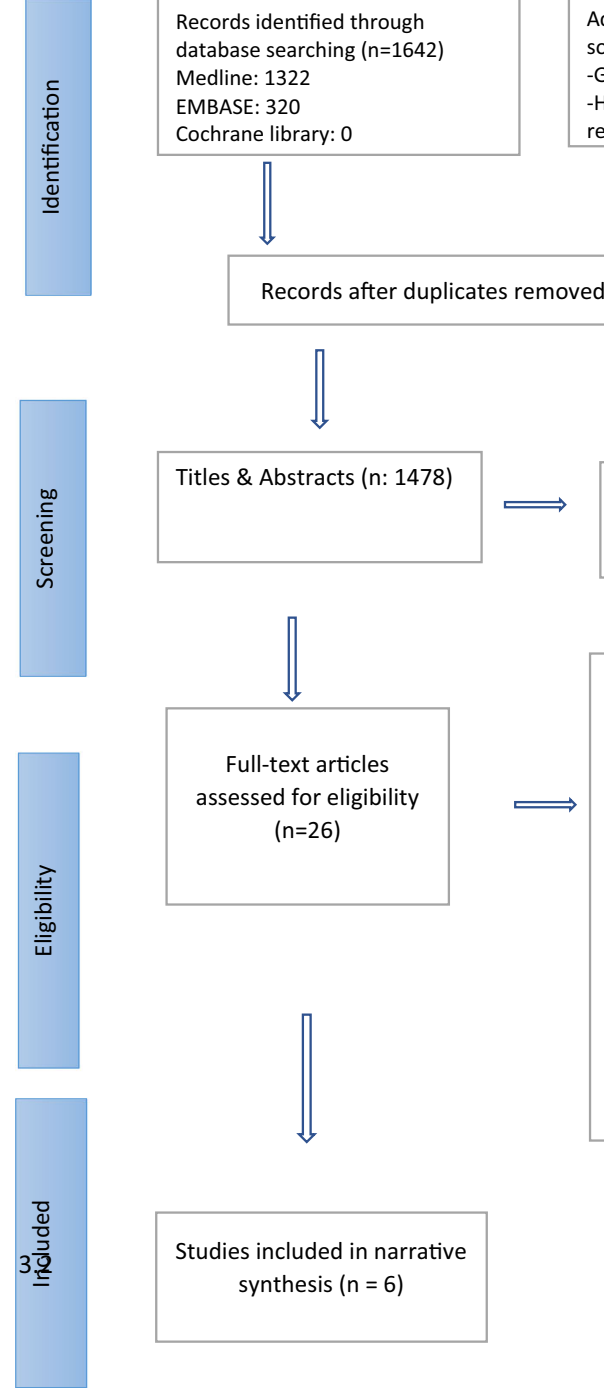

Additional records identified through other sources $(n=20)$

-Google scholar keyword searches

-Hand searches in the references lists of other

reviews

Full-text articles excluded, with reasons ( $n=20$ )

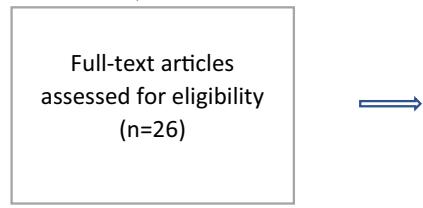

Type of studies: qual, unknown or combinations of uncontrolled quant, cross-sectional surveys \& qual $(N=5)$

No review of eHealth interventions $(N=4)$

Solely focusing on healthcare service outcomes $(N=5)$

Not reporting health/mental health outcomes $(N=3)$

Not focusing on population explicitly reported as ethnic minority or historically underserved $(N=2)$

Full-text non-retrievable $(N=1)$

Fig. 1 PRISMA flowchart of the selection process for systematic reviews PRISMA FLOW DIAGRAM

Two SRs (SR1, SR4) included only RCTs. SR3 also included studies that adopted mixed-methods studies, and four SRs (SR2, SR3, SR5, SR6) included studies that reported feasibility or pilot studies (Table 7). As the included SRs focused on designs other than RCTs, participant and trial data were not pooled together, and participant characteristics were organised by contexts, populations and areas of community wellbeing (Table 5).

All SRs included studies focusing on ethnic minority and/or historically underserved populations in the USA. Two SRs (SR5, SR6) included multi-national samples of participants residing in predominantly in developed countries. Among the studies done within the USA, the majority of participants were African Americans or mix African American and/or ethnic minorities residing in urban/metropolitan areas. There were 
Table 3 Details for Included SRs

\begin{tabular}{|c|c|c|c|}
\hline SRs & Author & Title & Date \\
\hline SR1 & $\begin{array}{l}\text { Heitkemper, Mamykina, Travers \& } \\
\text { Smaldone }\end{array}$ & $\begin{array}{l}\text { Do health information technology self-management in- } \\
\text { terventions improve glycaemic control in medically } \\
\text { underserved adults with diabetes? A systematic review } \\
\text { and meta-analysis }\end{array}$ & 2017 \\
\hline SR2 & $\begin{array}{l}\text { Bennet, Steinberg, Stoute, Lanpher, } \\
\text { Lane, Askew, Foley \& Baskin }\end{array}$ & $\begin{array}{l}\text { Electronic health (eHealth) interventions for weight man- } \\
\text { agement among racial/ethnic minority adults: a sys- } \\
\text { tematic review }\end{array}$ & 2014 \\
\hline SR3 & $\begin{array}{l}\text { Anderson-Lewis, Darville, } \\
\text { Mercado, Howell, \& Di Maggio }\end{array}$ & $\begin{array}{l}\text { mHealth Technology Use and Implications in Historically } \\
\text { Underserved and Minority Populations in the United } \\
\text { States: Systematic Literature Review }\end{array}$ & 2018 \\
\hline SR4 & Baptist, Islam, \& Joseph & $\begin{array}{l}\text { Technology-based interventions for asthma - can they help } \\
\text { decrease health disparities? }\end{array}$ & 2016 \\
\hline SR5 & $\begin{array}{l}\text { Ruggiano, Brown, Li, \& } \\
\text { Scaccianoce }\end{array}$ & Rural caregivers and technology: What is the evidence? & 2018 \\
\hline SR6 & Sinclair & $\begin{array}{l}\text { Effectiveness and user acceptance of online chronic } \\
\text { disease management interventions in rural and remote } \\
\text { setting: systematic review and narrative synthesis. }\end{array}$ & 2015 \\
\hline
\end{tabular}

fewer studies focusing solely on Latino and Hispanic populations and an even fewer focusing on other ethnic (e.g. Korean, Asian Pacific) and or underserved populations (e.g. rural or low socio-economic status, or both). Among the SRs that included multinational samples, the majority of them were from the USA. However, the multinational samples did include samples from other European countries including the Netherlands, Germany, Norway, UK, Spain, and Greece, as well as samples from Australia. Two of the multi-national SRs included three samples from Asia (China and Korea). The populations of multinational SRs were ethnic minorities and rural or remote populations. All six SRs addressed a range of public health topics of interest for community well-being such as diabetes, weight management, chronic conditions management, asthma, caregiver burden and stress.

\section{Narrative Synthesis of Results}

All SRs reported studies describing eHealth or mHealth interventions delivered online. The SRs (Table 6) described computer and mobile-based interventions with and without internet access, as well as interventions delivered via other technological devices. SR2, SR4, SR5 and SR6 reviewed interventions with features that allowed online interactions with peers and other users. All SRs included either online or other forms of interaction with clinical and non-clinical staff. The degree of interaction ranged from one-way sharing of information (e.g. automated text-messaging and reminders; tracking patient data; tracking of mobile use, sharing of treatment plans) to interactive forms of communication (e.g. online delivery of educational modules, unlimited access to interactivebased interventions, e-mail communication, online counselling.

SR2 and SR6 reviewed studies that were solely delivered via web-based applications (e.g. smartphones, websites, portals, interactive online groups, videoconferencing). SR2 reported six studies, five of which were randomised controlled trials, that 


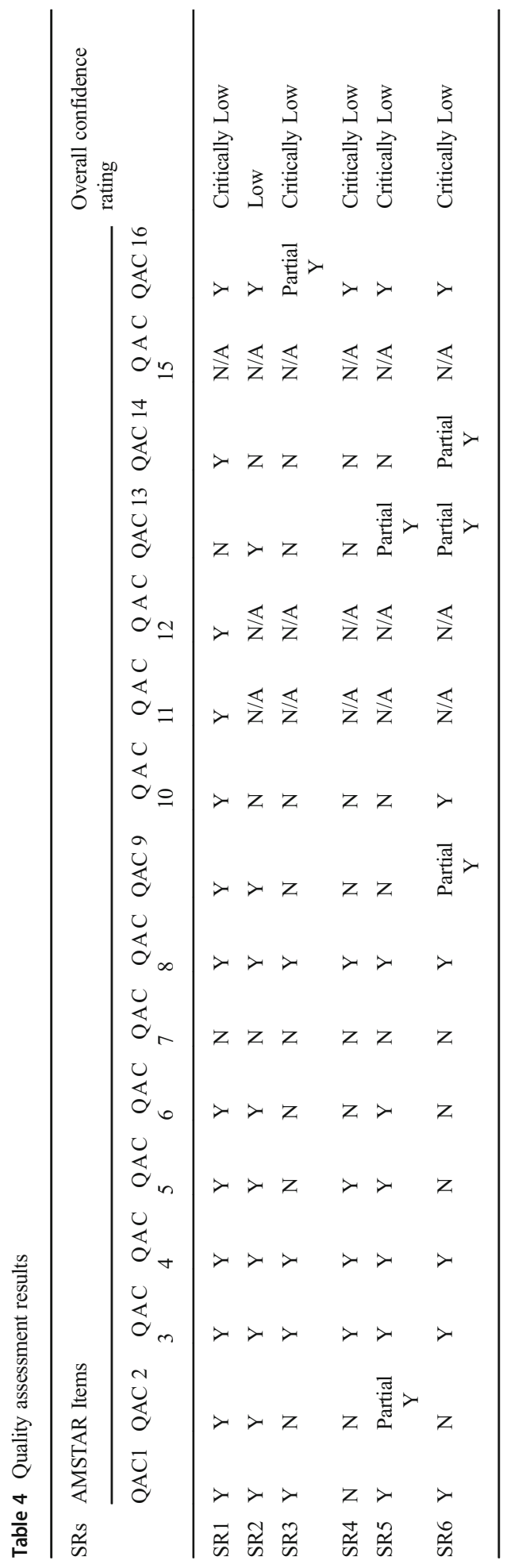


Table 5 SRs population characteristics

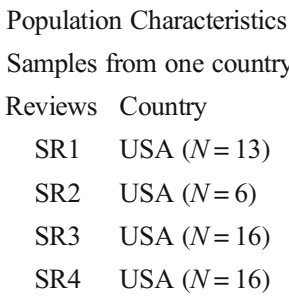

Community characteristics

Reviews Types of minority/underserved populations

SR1 Population demographics: medically underserved populations (at least $51 \%$ of the sample meeting federal poverty guidelines or racial/ethnic minorities or residing in a rural area),

-Included studies: underserved/ethnic minorities demographics and samples: Age (mean): 55, Gender: $66 \%$ female, $74 \%$ Ethnic minorities majority African Americans

SR2 Population demographics: racial/ethnic minority populations

-Demographics: At least 50\% racial/ethnic minority adults (or study outcomes reported by race/ethnicity)

-Study samples: Black \& Hispanic $(N=3)$, Black $(N=2)$, Black, Hispanic, Asian Pacific Islander \& Unknown $(N=1)$

SR3 Population demographics: a specific priority population group or subgroup (underserved populations)

- Included studies: underserved populations' demographics: urban/metropolitan, females, Age: 15-30

- Included studies: ethnic minorities samples: African American or mix African American and Latino or Hispanic $(N=9)$, Hispanic and Latino populations $(N=7)$, Korean American women $(N=1)$

SR4 Population demographics: black and urban/low-income individuals

- Included studies: underserved populations: demographics: Urban/inner city/low income/minority populations of all ages

- Included studies: ethnic minorities samples: African Americans

Well-being

Reviews Public Health Topic of Interest

SR1 Diabetes

SR2 Weight management

SR3 Technology \& health disparities (diabetes and sexual, reproductive, and maternal and child health; vaccinations and health; HIV and AIDS prevention for high-risk population groups)

SR4 Asthma

\section{MULTINATIONAL SAMPLES}

\section{Reviews Countries}

SR5 USA $(N=17)$, Netherlands $(N=1)$, Germany $(N=3)$, Norway $(N=1)$ Canada $(N=1)$, Multinational (UK, Spain, Greece) $(N=1)$, China $(N=2)$

SR6 USA $(\mathrm{N}=7)$, Canada $(N=2)$, Australia $(N=2)$, Korea $(\mathrm{N}=1)$, American military stations in Western Pacific locations $(\mathrm{N}=1)$

Community Characteristics

Reviews Types of minority/underserved populations

SR5 Population demographics: rural ethnic minority populations (non-rural not excluded)

- Included studies: underserved population demographics: $10 \%(\mathrm{~N}=3)$ rural dwellings (Canada, Norway and New England (USA)

- Included studies: ethnic minorities samples: specific cities $(n=9)$, State-wide \& Multi-state sampling $(N=9)$, unspecified geography $(N=4)$ 
Table 5 (continued)

SR6 Population demographics: rural (non-metropolitan and non-urban) or remote locations

- Included studies: underserved populations' demographics: adults with specific chronic disease group $(N=7)$, patients with a broad range of chronic diseases $(N=6)$, women with chronic disease $(N=3)$, children with chronic disease $(N=3)$

- Included studies: ethnic minority samples: Of the studies conducted in United States, Canada, and Australia, participants were predominantly of Caucasian ethnic backgrounds.

Well-being

Reviews Public Health Topic of Interest

SR5 Caregiver burden and stress

SR6 Chronic physical disease

described online interventions (e.g. website information and interactive voice-activated technology) or combinations of web-based material, and online provider-mediated interventions (e.g. online nutrition education, counselling via e-mail, biobehavioural internet treatment). SR6 reviewed thirteen studies, three of which were randomised controlled trials. The eHealth or mHealth interventions reviewed in SR6 consisted of web-based self-management interventions, (e.g. access to specific web-sites or webpages), interactive online-groups (e.g. interactive bulletin board, online support groups), and provider-mediated interventions (e.g. remote teleconsultation, nursesupported use of online health information; online personalised diabetes management education).

Two SRs (SR1 and SR3) reported studies that delivered interventions using computer-based and mobile-based technology that either required access to the internet or access to a mobile phone. SR1 reviewed thirteen studies of eHealth and mHealth interventions delivered via web-based applications (e.g. videoconferencing, computerbased multimedia lessons) or computer software programmes and mobile technology that did not require access to the internet. Many of those interventions in SR1 were delivered via a combination of follow-up calls, group sessions or face-to-face training. SR1 consisted of five trials. SR3 reviewed sixteen studies about interventions delivered via mobile phones only. The delivery of the interventions reviewed in SR 3 involved diverse ranges of access from limited minutes on a device to extensive internet access. The simplest intervention in SR3 required very limited minutes availability and phone

Table 6 Intervention delivery methods

\begin{tabular}{llll}
\hline $\begin{array}{l}\text { Web-based applications (e.g. smartphones, } \\
\text { websites, portals, interactive online groups, } \\
\text { videoconference) }\end{array}$ & $\begin{array}{l}\text { Computer, Cell phones } \\
\text { (offline) (e.g. software } \\
\text { programmes, texting) }\end{array}$ & $\begin{array}{l}\text { Other (e.g.MP3, } \\
\text { DVD/videotapes, } \\
\text { videogames) }\end{array}$ \\
\hline SR1 & $\mathrm{x}$ & $\mathrm{x}$ & \\
SR2 & $\mathrm{x}$ & $\mathrm{x}$ & $\mathrm{x}$ \\
SR3 & $\mathrm{x}$ & $\mathrm{x}$ & $\mathrm{x}$ \\
SR4 & $\mathrm{x}$ & $\mathrm{x}$ & $\mathrm{x}$ \\
SR5 & $\mathrm{x}$ & & \\
SR6 & $\mathrm{x}$ & & \\
\hline
\end{tabular}


features, while most of the reported interventions required texting and calling capabilities (e.g. just-in-time tips, health promotion messages, phone counselling, remote nurse support). Two interventions in SR3 required a mobile phone allowing interactive voice response (IVR) system capability to support self-monitoring and patient symptom monitoring. One intervention in SR3 required access to Facebook and another required streaming online videos on a smartphone combined with online questionnaires.

SR4 and SR5 reported studies describing electronic (e.g. computer-based and mobile-based interventions) as well as other technology-based interventions (e.g. MP3s, DVDs, videotapes, videogames). SR4 reviewed sixteen interventions for asthma education, asthma monitoring and management. The interventions in SR4 (three feasibility studies and 13 RCTs) were delivered via computer software and laptops available in clinics, school settings or home visits using MPEG audio layer-3 players, CR-ROMs, asthma education video games on Super Nintendo console, and mobile phones allowing texting and supporting interactive voice response. The technology used in the interventions in SR4 determined characteristics of the content of the interventions. For example, the Health Buddy intervention written about by Guendelman et al. 2002 and included in SR4 was more interactive, allowing greater direct communication with individual patients. SR5 reviewed thirty studies (twenty-one RCTs, two control trials with partial randomisation, and seven quasi-experimental pilot studies) reporting interventions for caregiver burden and stress. The interventions in SR5 lasted between thirty days and eighteen months and were delivered via basic telephone, websites, web or videoconferencing, and videos. SR5 telephone-based interventions were more widely utilised $(N=15)$ as the required technology has been available since the $90 \mathrm{~s}$, compared to smartphone or video phone technologies that became more widely available in the 2000 s.

\section{Outcomes of the Narrative Synthesis}

Five SRs used narrative synthesis to synthesize findings on the effectiveness of eHealth and mHealth interventions in improving a range of physiologic and psychosocial outcomes and self-healthcare management, while one SR performed a meta-analysis. Four SRs (SR1, SR2, SR4, SR6) included studies that reported an improvement in physiologic outcomes. Three SRs (SR1, SR5, SR6) included studies reporting improvements in psychosocial outcomes. Four SRs (SR1, SR3, SR4) included studies reporting improvement in health-related knowledge and self-care management (Table 8). However, only three SRs (SR1, SR2, SR5) included the use of a specific appraisal tool and rating as part of the quality assessment process (Table 7).

SR1 reported a meta-analysis of health information technology (HIT) diabetes selfmanagement (DSM) education interventions. The meta-analysis in SR1 also showed that internet-based interventions were the most effective interventions with the greatest effectiveness in both 6 months and 12 months intervals. SR1's review of HIT DSM education interventions also demonstrated various physiologic benefits.

SR2 found that eHealth weight management intervention can have short-term success among racial/ethnic minority groups, while SR3 showed that mHealth technologies had the potential to influence behaviours among historically underserved and ethnic minority populations especially through the delivery of health education messages via SMS text messages. SR4 showed that technology-based asthma interventions 


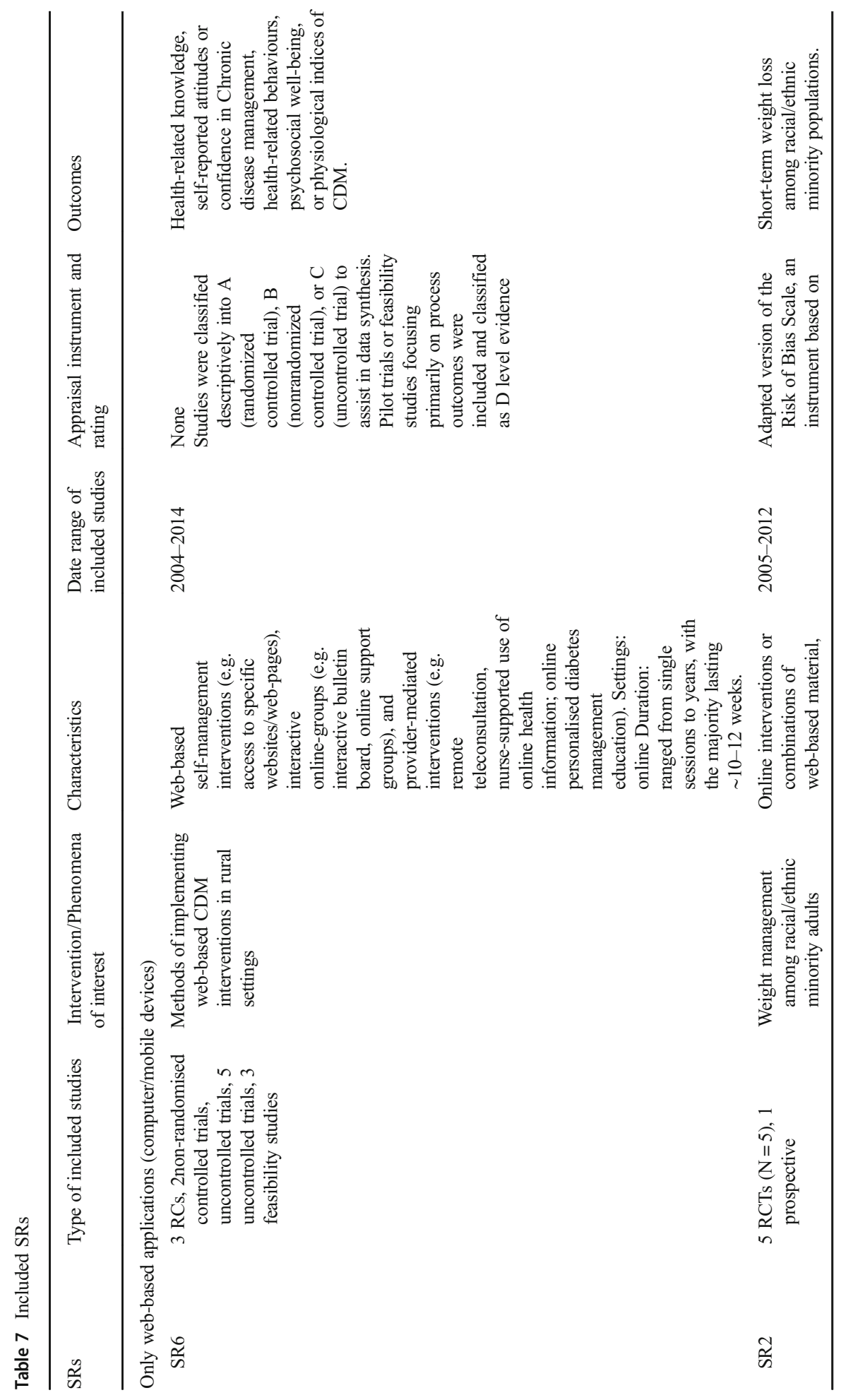




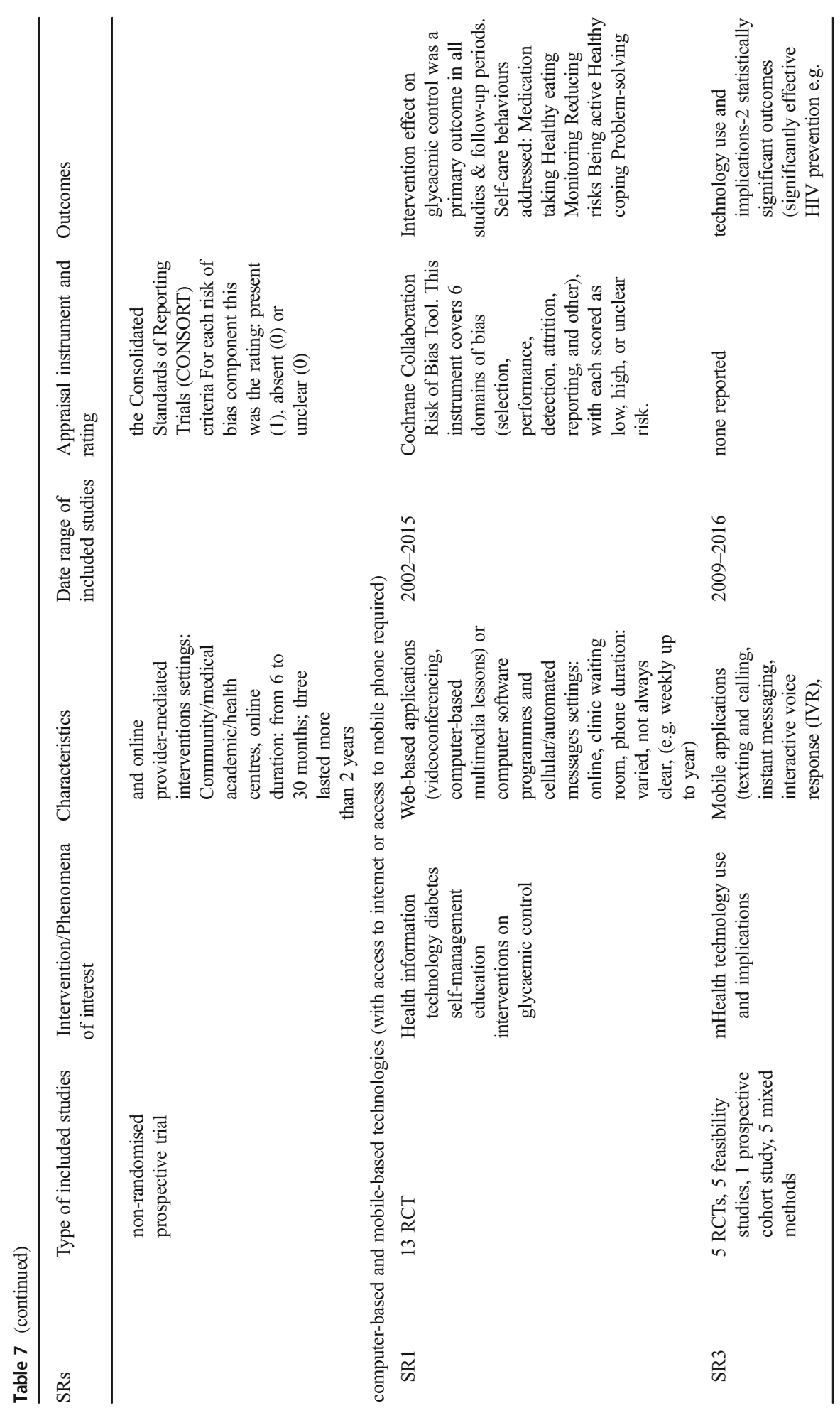




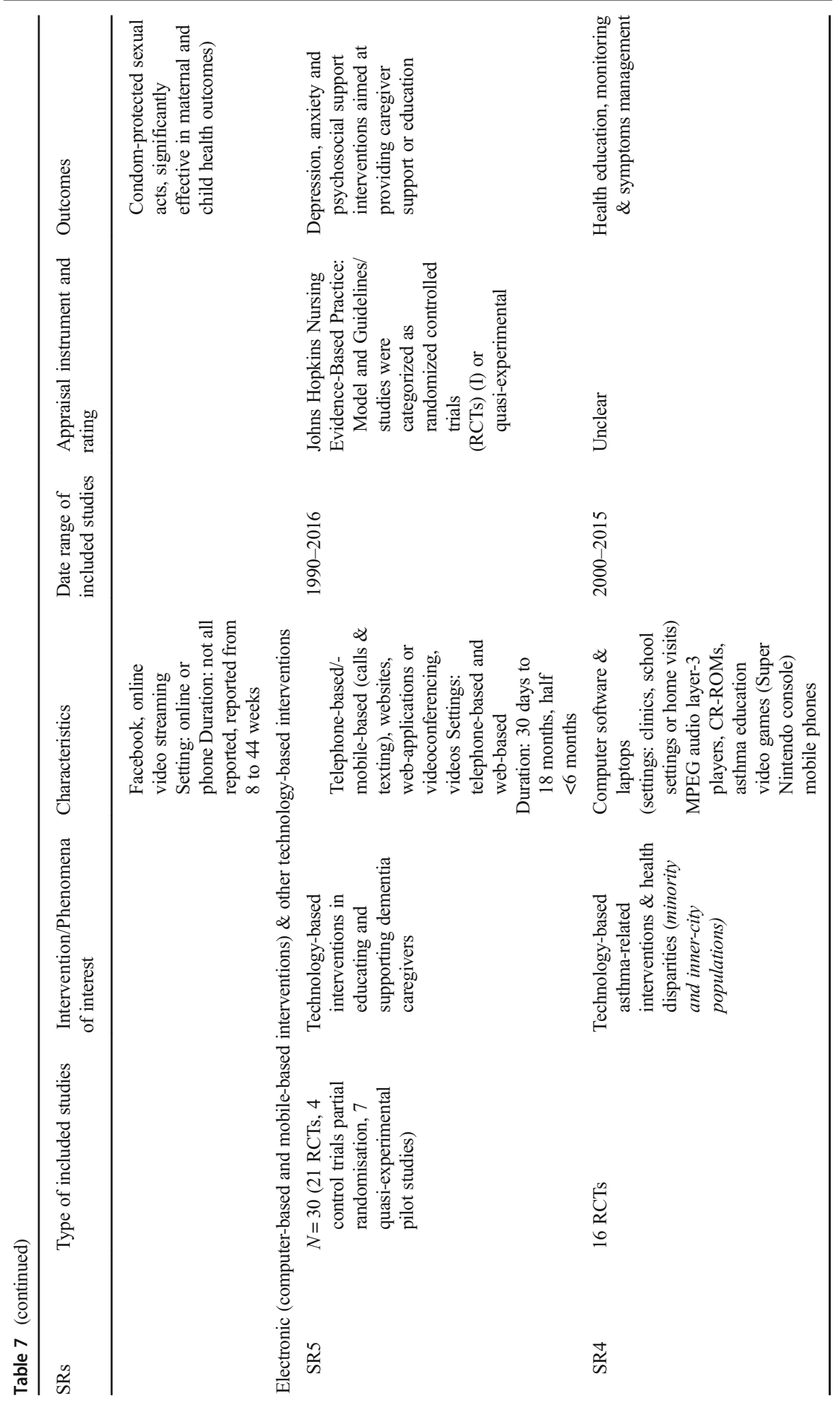




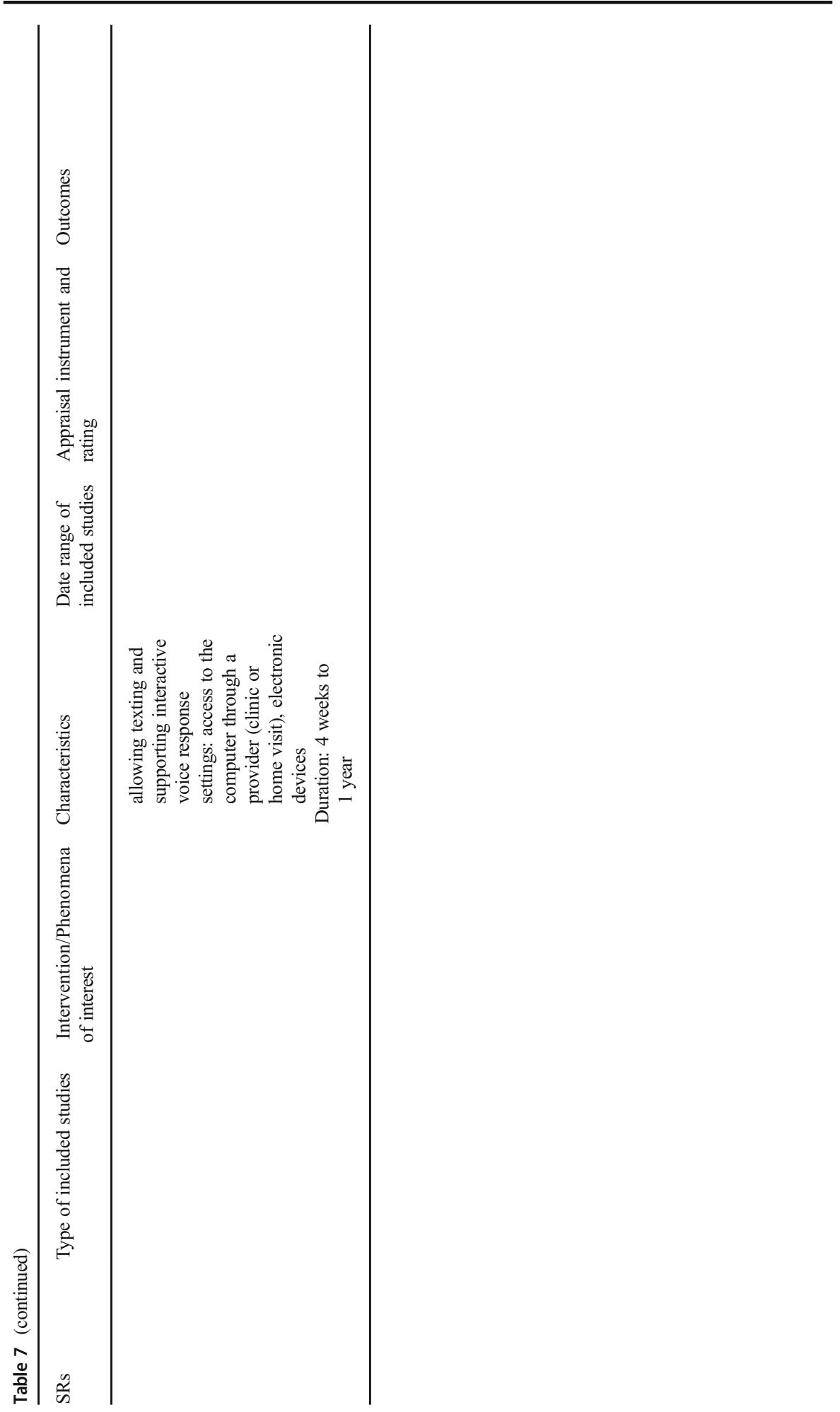

暨 Springer 
have been more frequently successful in improving asthma knowledge, and medication adherence. SR 4 also indicated that computer and web-based interventions have been successful in improving self-reported symptoms and functional status, and one intervention delivered via Nintendo consoles and one via interactive voice response (IVR) showed significant improvement in participant reported quality of life.

SR5 was the only SR that solely reported on psychosocial outcomes of technologybased interventions. In particular, SR5's review of thirty phone-based, video-based, computer-based or web-based interventions tested with community-based care-givers demonstrated that there is some evidence for decreasing depression and anxiety, while in the studies where the rural populations were clearly identified, there was an improvement in perceived emotional and social support. Finally, SR6 reported a review of web-based self-management interventions, interactive online groups, and providermediated interventions showing that eHealth interventions can substantially increase participant psychosocial adaptation and knowledge of chronic disease management strategies (Table 8).

\section{Tailoring of Interventions}

Although all SRs included studies that reported eHealth or mHealth interventions delivered to ethnic minorities or historically underserved populations, only some of SRs (SR1, SR2, SR3, SR4, SR6) reported studies tailored to a target population (Table 9). SR2 assessed the degree to which trials were optimised to their target population. SR2 found that only two out of six trials adopted specific tailoring strategies. One of the studies, conducted by Bennett et al. (2012) optimised intervention content for lower literacy capabilities or other sociocultural characteristics. The other, conducted by Hollis et al. 2008 incorporated consultation with external committees to maximise cultural optimisation during the development stage of an intervention. SR6 reported three feasibility studies that described specific tailoring strategies. In SR6, one feasibility study conducted by Jernigan and Lorig (2011) was culturally optimised for its target population (American Indian/Alaskan Native peoples) while two other feasibility studies, one conducted by Weinert and Hill (2005) and the other by Guilcher et al. (2013) assessed the acceptability and feasibility of interventions in rural USA and Canada. Although eight of ten of the studies reported attrition rates in SR6, none reported optimisation techniques.

SR1 produced a review of thirteen diabetes interventions, eight of which were tailored to their target population in terms of language, literacy, and culture. Seven interventions in SR1 were available in a second language (Spanish, Cantonese) and three interventions were optimised to low-literacy levels and cultural dimensions. One intervention (a healthcare staff) was trained in improving communication with lowliteracy population in SR1, and one was optimised to the user's level of computer experience.

SR3 included two studies that were tailored to the language of their target population groups and two that were tailored to individual participant responses (e.g. diet preferences, food consumption) but no tailoring strategies were reported in relation to cultural characteristics and literacy level. The reviewers in SR3 note that a characteristic of one of the most notable mHealth studies in the USA, conducted by Evans et al. (2012) is that it utilised culturally tailored health messages, called text4baby, along with an in- 


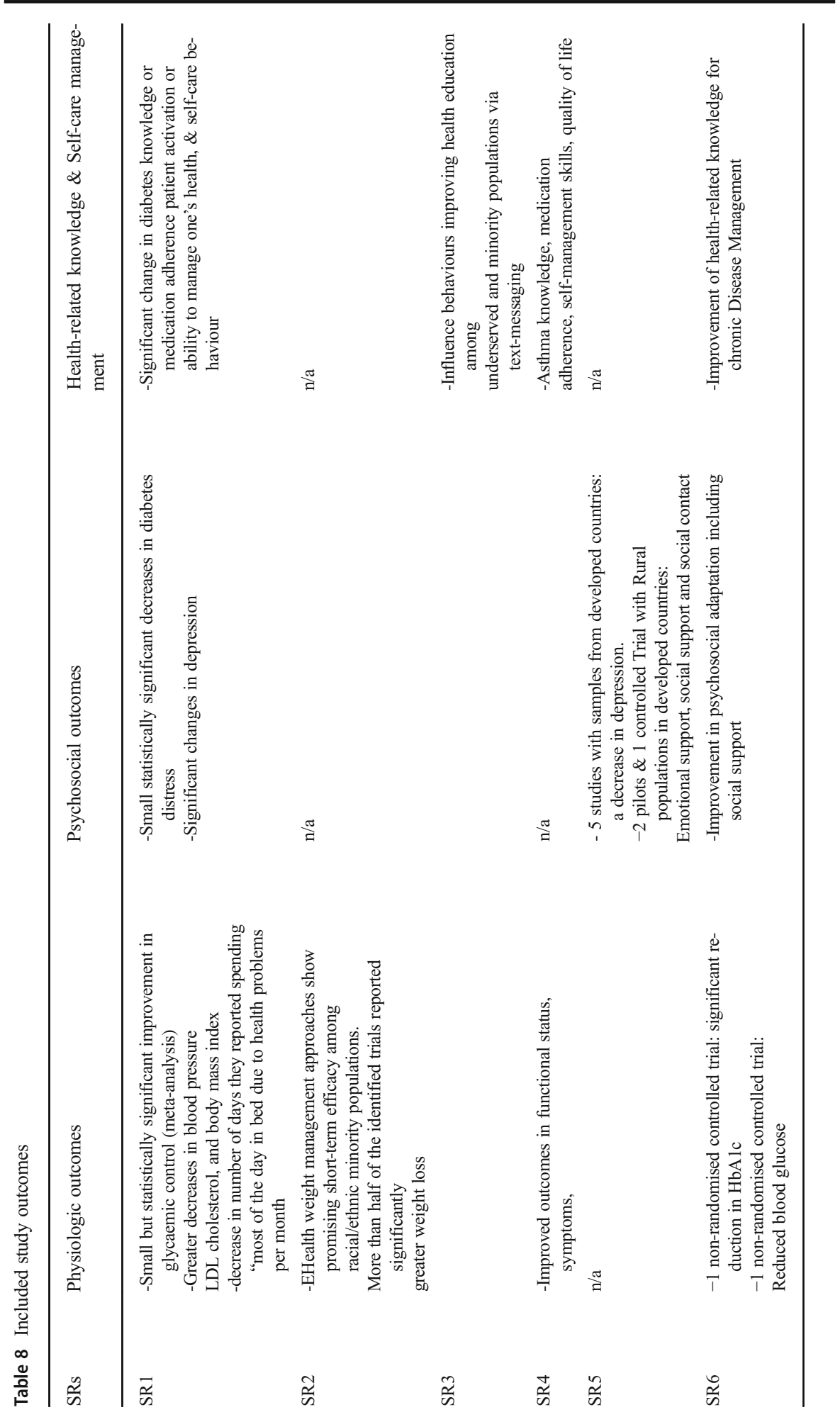


Table 9 Included studies reported tailoring processes

Studies Tailoring strategies

SR1 8/13 interventions used tailoring strategies focusing on language, literacy, and culture and some focused on technology usability (e.g. participant numeracy and psychomotor skills), 3/4 that delivered education modules incorporated tailored materials or communication to their specific populations

SR2 Two studies reported tailored interventions to racial/ethnic minority participants (e.g. literacy capabilities \& sociocultural characteristics)

SR3 Two interventions tailored to participant language

SR4 9/13 interventions had tailored content

SR5 n/a

SR6 Studies examined user acceptance and feasibility 1 study only optimized for American Native/Alaskan native peoples 1

depth investigation to attain optimal methods for intervention delivery to its target population.

SR4 was the review with the highest number of interventions containing tailored content to target populations. Tailoring strategies in SR4 included offering participants the capacity to record their own voice messages or form text messages, tailoring content to personal, attitudinal, and disease-specific characteristics and involving participants in the delivery of content to increase acceptability of an intervention and reduce stigma. SR5 did not report whether interventions adopted tailoring strategies and patient engagement in 17 out of 30 studies.

\section{Discussion}

This umbrella review identified and assessed SRs of intervention studies relating to the effectiveness of eHealth and mHealth interventions targeting the health or mentalhealth outcomes of ethnic minority and/or historically underserved populations in developed countries. For this reason, it only included SRs that met those specific population criteria, reviewing patient-centred interventions and reported on their health or mental-health related outcomes.

\section{eHealth and mHealth Technology Use}

All SRs included studies delivered online. Only two SRs (SR2 and SR6) focused solely on web-based interventions. This can be explained by the limited evidence-base from which conclusions on the effectiveness of web-based interventions are often drawn and the low quality of that evidence (Bennett et al. 2014; Cotter et al. 2014). Interventions delivered via mobile phones, often with simple features such as text messaging and without requiring access to internet, were the second most frequently reviewed type of interventions. That is in accordance with findings on trends in technology use and ownership and variations over time, such as reduction in the use of landline phones (Blumberg and Luke 2014; Anderson 2015), which indicate that ethnic minorities 
(African Americans and Hispanics) may be more likely than Caucasians to own mobile devices.

Five out of six of the SRs (SR1, SR2, SR3, SR4, SR6) reported improvements in participant physical health or self-care management skills, while only three SRs (SR1, SR5, SR6) reported improvement in a few psychosocial and mental health outcomes. This is in accordance with the challenges associated with recruiting minority populations for clinical trials or for mental health research, as found by Mason et al. (2003) as well as Waheed et al. (2015). Our analysis suggests that there is evidence supporting the effectiveness of web-based interventions improving ethnic minority and historically underserved community's health, ranging from weight management, asthma and diabetes knowledge and medical adherence.

\section{eHealth, mHealth and Community Health Inequalities}

All SRs included ethnic minority and underserved populations within the USA, and only two SRs (SR5 and SR6) included international samples from other developed countries. Within the USA, the majority of the SRs focused more on African Americans, whereas for SRs including international samples, minority and underserved populations focused on different aspects of demographics (e.g. rurality) and medical conditions (e.g. chronic illness). The SRs in this umbrella review revealed small variation in the minority and underserved populations.

Our analysis suggests tackling community health inequalities requires interventions demonstrating cultural sensitivity. This can mean designing and evaluation interventions through sampling procedures that are representative of the characteristics of communities. Examples can be the improvement of representation of minorities with a country (e.g. Chinese, Korean, Mexican, Native Americans, etc.), as found by Roosa et al. (2008) or in different countries such as the South Asian populations in UK, or North Africa populations in central Europe as found by Khan et al. (2019) as well as Waheed et al. (2015). Previous research highlights the importance of engaging communities in the research process in order to improve the quality of care (Holzer et al. 2014; Eyles et al. 2016). It has been suggested that recruitment and development of eHealth and mHealth interventions should be designed to systematically address the health inequalities of a wider range of minorities or underserved populations in a community (e.g. transgender people, refugees, substance users, people living with HIV, homeless) (Bonevski et al. 2014; Ellard-Gray et al. 2015; Horowitz et al. 2009).

\section{Content Tailoring Strategies to Community Characteristics}

The content of the interventions reported in the SRs varied substantially, ranging from health education information (e.g. diabetes knowledge, asthma knowledge, selfmanagement skills, etc.) to teleconsultations. Tailoring strategies described in the SRs involved intervention content optimised to target population language and sociocultural characteristics as well as tailoring to individual literacy, numeracy skill level and psychomotor capabilities. In three SRs (SR1, SR3, AND SR5) tailoring was achieved through usability testing and evaluation of participant preferences in order to increase intervention acceptability. However, few interventions delivered to minority and 
underserved populations contained content that was specifically tailored to these populations. In particular, there was not an SR that solely reported studies that adopted tailoring strategies, while only two SRs (SR1 and SR4) included such studies in the majority (eight out of thirteen and nine out of thirteen respectively). SR1 concluded that interventions that incorporated tailoring strategies were the most successful ones.

One key finding of this umbrella review was the limited extent to which evidence is drawn from studies with specific tailoring strategies for the delivery or evaluation of eHealth and mHealth interventions to their target population. This finding concurs with recent SRs on the effectiveness of health promotion interventions in minority or underserved populations indicating that linguistic and cultural tailoring can increase effectiveness (e.g. improve diabetes prevention, promotion of physical activity) and engagement (Lagisetty et al. 2017; Mendoza-Vasconez et al. 2016).

\section{Quality and Relevance of this Umbrella Review}

The SRs were found to have low to critical low quality as assessed by AMSTAR 2. However, our findings are relevant and important because eHealth interventions are gaining popularity for addressing mental and physical well-being and our findings offer direction for the development of guidelines to deliver quality interventions to improve ethnic minority and historically underserved population's well-being at a time when such is needed.

\section{Implications for Community Organizers, Policymakers and Researchers}

This umbrella review was conducted with an aim to provide guidance to community organizers and policy makers seeking to address health inequalities through eHealth and mHealth interventions. A rigorous procedure was applied indicating that the SRs used in this umbrella review represent the best available SRs and the findings in this umbrella review are the most relevant to date. The authors suggest that there is a great need for the identification and development of quality criteria, as the development and use of eHealth and mHealth interventions is growing (Marcolino et al. 2018). Analysis of the findings of this umbrella review yielded the following criteria for policy makers and community organizers engaged in eHealth and mHealth intervention policies or programs:

- eHealth and mHealth interventions should require a rigorous examination of the characteristics of diverse minority and socially disadvantaged populations, their acceptability of the intervention content and the suitability of the technological platforms.

- Target populations should to be included in each stage of the development and evaluation of eHealth and mHealth interventions.

- Future umbrella reviews should incorporate qualitative and mixed methods reviews as well as reviews of grey literature, while quality assessment methods of those reviews should also accordingly reflect and acknowledge the context of performing evaluation research with minority or underserved populations

- Future studies and SRs of the effectiveness of eHealth and mHealth interventions for ethnic minority and historically underserved populations should strive to 
provide useful guidance to policymakers, academics, and industry professionals towards delivering community-wide digital interventions. Future research could be benefited by the adoption of theory-driven, person-centred approaches. Such approaches should follow co-design processes in the development of relevant and feasible eHealth interventions (Yardley et al. 2015; Walsh et al. 2019).

To the last recommendation, the advantage of a co-design process is that it can address the external context of an intervention (Kemm 2006) by incorporating context parameters in process evaluations, complementing short-comings of evidence-based research that often views the body of evidence as being a shared experience of reality (Huxley et al. 2015; Chou et al. 2013). Such research can incorporate the living experiences of ethnic minority, marginalised, and economically and/or medically underserved populations, hence, its outputs can benefit greatly community well-being.

\section{Strengths and Limitations}

Strengths of this umbrella review included the systematic search for SRs following a previously developed and registered protocol, the report of the findings using the PRISMA guidelines, and the use of AMSTAR 2 for quality assessment. We included only SRs published in peer-reviewed journals to maximise the rigor of the evidence. We included SRs that targeted either ethnic minority or historically underserved populations (or both) without applying strict socio-economic or health criteria, thus, allowing for an inclusion of diverse economic, geographic, or medically underserved populations.

A limitation of this umbrella review is the low quality of the included SRs. According to the results of our quality assessment, this was due to the limited use of quality assessment procedures undertaken or a lack of discussion about the issue of quality and quality assessment in the SRs.

Some of those limitations may reflect the challenges in designing and evaluating interventions that target minority or underserved populations. Another limitation of this umbrella review was that due to time and resources constraints the searches were limited to four academic databases and only peer-reviewed articles published in the English language. As grey literature and unpublished reviews were excluded (e.g. conference proceedings, unpublished theses) informative evidence may have been missed. Moreover, as we did not apply any strict definition to the term historically underserved populations, we did not specifically aim to include studies that target difficult to reach or medically under-researched populations. Finally, considering that the current review assessed only six intervention SRs published in English, and it may be concluded that there might be more SRs to assess in relation to the topic examined here published in other languages as well as those published after the time of this umbrella review. For example, a SR that was published a few months after the completion of the present umbrella review, and reviewed the evidence on digital mental health interventions for marginalised and underserved populations (Latin and African Americans, rural populations, individuals experiencing homelessness, and sexual and gender minorities) concluded that there is emerging evidence for the feasibility and acceptability of those interventions but there is a lack of evidence on their efficacy on a 
large-scale (Schueller et al. 2019). Those findings offer a clear description of the state of the evidence in the field applying specific quality criteria that can inform the focus of future SRs and assist policymakers to develop evidence-based policies.

This umbrella review, drawing from the findings of SRs published as of March 2019, offers a clear overview of the effectiveness of eHealth and mHealth interventions among historically underserved populations in developed countries. It highlighted areas for which there is stronger evidence for their effectiveness (i.e. web-based interventions for the improvement of health behaviours) as well as those areas for which there is limited evidence-base (e.g. delivery and evaluation of interventions tailored to communities' characteristics).

\section{Conclusion}

An aim of this umbrella review was to provide evidence-based criteria for the premise of patient-centred eHealth and mHealth interventions for mending inequalities in healthcare for communities and promoting community well-being. Our analysis indicates that there is a growing evidence on the effectiveness of eHealth and mHealth interventions targeting specific physiologic (e.g. improvement in glycaemic control and weight management) and psychosocial outcomes (e.g. improvement in psychosocial adaptation) as well as health-related knowledge and self-care management among historically underserved populations in developed countries. Furthermore, it is evident that mHealth interventions may be particularly effective, while efforts need to be made to customize the interventions to ensure equitable and equal access and delivery of them to minority and historically underserved populations.

This umbrella review identified strategies that may minimise the influence of social health inequalities while maximising the effectiveness of those interventions to their target populations. It is evident from this umbrella review that efficient strategies for the purpose of providing culturally sensitive interventions involves the meticulous tailoring of interventions to the target audience by including the target audience in the research and development process, as well as making sure that a community's underserved populations are adequately represented in any sampling and implementation procedures. We conclude that rigorous methods and reporting are essential to affirm transparency and reliability for all relevant parties (e.g. clinicians, patients, funders) and develop a trusting relationship with the most vulnerable members of a community and increase their inclusion in the research process for the equal and equitable delivery and use of eHealth and mHealth interventions.

Funding Information This research received no external funding.

\section{Compliance with Ethical Standards}

Conflict of Interest The authors declare no conflict of interest. 
Open Access This article is licensed under a Creative Commons Attribution 4.0 International License, which permits use, sharing, adaptation, distribution and reproduction in any medium or format, as long as you give appropriate credit to the original author(s) and the source, provide a link to the Creative Commons licence, and indicate if changes were made. The images or other third party material in this article are included in the article's Creative Commons licence, unless indicated otherwise in a credit line to the material. If material is not included in the article's Creative Commons licence and your intended use is not permitted by statutory regulation or exceeds the permitted use, you will need to obtain permission directly from the copyright holder. To view a copy of this licence, visit http://creativecommons.org/licenses/by/4.0/.

\section{References}

\section{*SRs included in the umbrella review}

Adashi, E., Geiger, H., \& Fine, M. (2010). Health care reform and primary cared - the growing importance of the community health center. The New England Journal of Medicine, 362(22), 2047-2050. https://doi. org/10.1056/NEJMp1003729.

Anderson, M. (2015). Technology device ownership: 2015. Pew Research Center. Retrieved from https://www.pewinternet.org/2015/10/29/technology-device-ownership-2015/[Online Resource].

*Anderson-Lewis, C., Darville, G., Mercado, R., Howell, S., \& Di Maggio, S. (2018). mHealth technology use and implications in historically underserved and minority populations in the United States: systematic literature review. JMIR mHealth and uHealth, 6(6), 2047-2050. https://doi.org/10.2196/mhealth.8383.

Arak, P. \& Wójcik, A. (2017). Transforming eHealth into a political and economic advantage, Polityka Insight, Warszawa. Retrieved from https://ec.europa.eu/digital-single-market/en/news/transformingehealth-political-and-economic-advantage [Online_Resource].

Aranda-Jan, C., Mohutsiwa-Dibe, N., \& Loukanova, S. (2014). Systematic review on what works, what does not work and why of implementation of mobile health (mHealth) projects in Africa. BMC Public Health, 14(188). https://doi.org/10.1186/1471-2458-14-188.

Aromataris, E., Fernandez, R., Godfrey, C. M., Holly, C., Khalil, H., \& Tungpunkom, P. (2015). Summarizing systematic reviews: methodological development, conduct and reporting of an umbrella review approach. International Journal of Evidence-Based Healthcare, 13, 132-140.

Aromataris, E., Fernandez, R., Godfrey, C.M., Holly, C., Khalil, H., \& Tungpunkom, P. (2017). Chapter 10: Umbrella reviews. In E. Aromataris \& Z. Munn (Eds.), Joanna Briggs Institute Reviewer's Manual. Retrieved from https://reviewersmanual.joannabriggs.org/ [Online_Resource].

*Baptist, A. P., Islam, N., \& Joseph, C. L. (2016). Technology-based interventions for asthma - can they help decrease health disparities? Journal of Allergy and Clinical Immunology: In Practice, 4(6), 1135-1142. https://doi.org/10.2196/jmir.2925.

Bellet, C., \& Frijters, P. (2019). Big data and well-being. In Helliwell, J., Layard, R., \& Sachs, J. World Happiness Report. Retrieved from https:/worldhappiness.report/ed/2019/big-data-and-well-being/ [Online_Resource].

Bennett, G. G., Warner, E. T., Glasgow, R. E., Askew, S., Goldman, J., Ritzowoller, D. P., Emmons, K. M., Rosner, B. A., \& Colditz, G. A. (2012). Obesity treatment for socioeconomically disadvantaged patients in primary care practice. Archives of Internal Medicine, 172(7), 565-574.

*Bennett, G. G., Steinberg, D., Stoute, C., Lanpher, M., Lane, I., Askew, S., Foley, P., \& Baskin, M. (2014). Electronic health (eHealth) interventions for weight management among racial/ethnic minority adults: a systematic review. Obesity Reviews: An official Journal of the International Association for the Study of obesity, 15(4), 146-158.

Bharmal, N, Derose, K.P., Felician, M., \& Weden, M.M. (2015). Understanding the Upstream Social Determinants of Health. Santa Monica, CA: RAND Health. Retrieved fromhttps:/www.rand. org/content/dam/rand/pubs/working_papers/WR1000/WR1096/RAND_WR1096.pdf [Online_Resouce].

Blumberg, S.J., \& Luke, J.V. (2014). Wireless substitution: early release of estimates from the National Health Interview Survey, January-June 2018. Retrieved from https://www.cdc. gov/nchs/data/nhis/earlyrelease/wireless201812.pdf. [Online_Resource].

Bonevski, B., Randell, M., Paul, C., Chapman, K., Twyman, L., Bryant, J., Brozek, I., \& Hughes, C. (2014). Reaching the hard-to-reach: a systematic review of strategies for improving health and medical research with socially disadvantaged groups. BMC Biomedical Research Methodology, 14, 1-29. https://doi. org/10.1186/1471-2288-14-42. 
Chigona, W., Nyemba, M., \& Metfula, A. (2012). A review on mHealth research in developing countries. The Journal of Community Informatics. Retrieved from http://ci-journal.net/index.php/ciej/article/view/941 [Online_Resource].

Chou, W. S., Prestin, A., Lyons, C., \& Wen, K. (2013). Web 2.0 for health promotion: reviewing the current evidence. American Journal of Public Health, 103(1), 9-18. https://doi.org/10.2105/AJPH.2012.301071.

CIA (2007). International Organizations and Groups. World Factbook. Retrieved from https:/www.cia. gov/library/publications/the-world-factbook/appendix/appendix-b.html [Online_Resource].

Cotter, A. P., Duran, T. N., Agne, A. A., \& Cherrington, A. L. (2014). Internet interventions to support lifestyle modification for diabetes management: a systematic review of the evidence. Journal of Diabetes and its Complications, 28(2), 243-251.

Dahn, B., Woldemariam, A. T., Perry, H., Maeda, A., von Glahn, D., Panjabi, R., Merchant, N., Vosburg, K., Palazuelos, D., Lu, C., Simon, J., \& Pfaffmann, J. (2015). Strengthening Primary Health through Community Health Workers: Investment Case and Financing Recommendations, Report of the United Nations Secretary General's Special Envoy for Financing the Health MDG and for Malaria. Retrieved from: https://www.who.int/hrh/news/2015/CHW-Financing-FINAL-July-15-2015.pdf?ua=1 [Online Resource].

Ellard-Gray, A., Jeffrey, N. K., Choubak, M., \& Crann, S.E. (2015). Finding the hidden participant: solutions for recruiting hidden, hard-to-reach, and vulnerable populations. International Journal of Qualitative Methods, 1-10.

Evans, W. D., Wallace, J. L., \& Snider, J. (2012). Pilot evaluation of the text4baby mobile health program. BMC Public Health, 12, e1031. https://doi.org/10.1186/1471-2458-12-1031.

Eyles, H., Jull, A., Dobson, R., Firestone, R., Whittaker, R., Te Morenga, L., Goodwin, D., \& Ni Mhurchu, C. (2016). Co-design of mHealth delivered interventions: a systematic review to assess key methods and processes. Current Nutrition Reports, 5(3), 160-167.

Eysenbach, G. (2001). What is e-health? Journal of Medical Internet Research, 3(2), e20. https://doi. org/10.2196/jmir.3.2.e20.

Freimuth, V. S., \& Quinn, S. C. (2004). The contributions of health communication to eliminating health disparities. American Journal of Public Health, 94(12), 2053-2055.

Germanakos P., Mourlas C., \& Samaras G. (2005) A mobile agent approach for ubiquitous and personalized eHealth information systems; Proceedings of the Workshop on 'Personalization for e-Health' of the 10th International Conference on User Modeling (UM' 05). Retrieved form http://cgi.csc.liv.ac. uk/ floriana/UM05-eHealth/UM05-W6.pdf [Online_Resource].

Guendelman, S., Meade, K., Benson, M., Chen, Y. Q., \& Samuel, S. (2002). Improving asthma outcomes and self-management behaviors of inner-city children: a randomized trial of the health buddy interactive device and an asthma diary. Archives of Pediatrics \& Adolescent Medicine, 156, 114-120.

Guilcher, S. J. T., Bereket, T., Voth, J., Haroun, V. A., \& Jaglal, S. B. (2013). Spanning boundaries into remote communities: an exploration of experiences with telehealth chronic disease self-management programs in rural northern Ontario, Canada. Telemedicine Journal and E-Health: the official journal of the American Telemedicine Association, 19(12), 904-909.

*Heitkemper, E. M., Mamykina, L., Travers, J., \& Smaldone, A. (2017). Do health information technology self-management interventions improve glycaemic control in medically underserved adults with diabetes? A systematic review and meta-analysis. Journal of the American Medical Informatics Association: JAMIA, 24(5), 1024-1035.

Helliwell, J. F., Huang, H., \& Wang, S. (2019). World Happiness Report 2019. Sustainable Development Solutions Network. Retrieved from https://worldhappiness.report/ed/2019/changing-world-happiness/ [online_Resource].

Hoffman, A. (2019). Building on the inherent strengths of green space environments: promoting trust, democracy, and resilience among ethnically diverse groups. Journal of Prevention \& Intervention in the Community, 21, 1-15. https://doi.org/10.1080/10852352.2019.1625600.

Hollis, J. F., Gullion, C. M., Stevens, V. J., Brantley, P. J., Appel, L. J., Ard, J. D., Champange, C. M., Dalcin, A., Erlinger, T. P., Funk, K., Laferriere, D., Lin, P. H., Loria, C. M., Samuel-Hodge, C., Vollmer, W. M., \& Svetkey, L. P. (2008). Weight loss during the intensive intervention phase of the weight-loss maintenance trial. American Journal of Preventive Medicine, 35, 118-126.

Holzer, J. K., Ellis, L., \& Merritt, M. W. (2014). Why we need community engagement in medical research. Journal of Investigative Medicine, 62, 851-855.

Horowitz, C. R., Robinson, M., \& Seifer, S. (2009). Community-based participatory research from the margin to the mainstream: are researchers prepared? Circulation, 119(19), 2633-2642.

Huxley, C. J., Atherton, H., Watkins, J. A., \& Griffiths, F. (2015). Digital communication between clinician and patient and the impact on marginalised groups: a realist review in general practice. The British 
Journal of General Practice: The Journal off the Royal College of General Practitioners, 65(641), e813e821. https://doi.org/10.3399/bjgp15X687853.

Ioannidis, J. P. A. (2009). Integration of evidence from multiple meta-analyses: a primer on umbrella reviews, treatment networks and multiple treatments meta-analyses. Canadian Medical Association Journal, 181, $488-493$.

Jernigan, V. B. B., \& Lorig, K. (2011). The internet diabetes self-management workshop for American Indians and Alaska natives. Health Promotion Practice, 12(2), 261-270.

Kemm, J. (2006). The limitations of 'evidence-based' public health. Journal of Evaluation in Clinical Practice, 12(3), 319-324.

Khan, S., Lovell, K., Lunat, F., Masood, Y., Shah, S., Tomenson, B., \& Husain, N. (2019). Culturally-adapted cognitive behavioural therapy based intervention for maternal depression: a mixed-methods feasibility study. BMC Women's Health, 19(1), e21. https://doi.org/10.1186/s12905-019-0712-7.

Kohlhuber, M., Mielck, A., Weiland, S. K., \& Bolte, G. (2006). Social inequality in perceived environmental exposures in relation to housing conditions in Germany. Environmental Research, 101(2), 245-255.

Kontos, E., Blake, K. D., Chou, W. S., \& Prestin, A. (2014). Predictors of eHealth usage: insights on the digital divide from the Health Information National Trends Survey. Journal of Medical Internet Research, 16(7), e172. https://doi.org/10.2196/jmir.3117.

Koutras, C., Bitsaki, M., Koutras, G., Nikolaou, C., \& Heep, H. (2015). Socioeconomic impact of e-health services in major joint replacement: a scoping review. Technology and Health Care, 23(6), 809-817.

Lagisetty, P. A., Priyadarshini, S., Terrell, S., Hamati, M., Landgraf, J., Chopra, V., \& Heisler, M. (2017). Culturally targeted strategies for diabetes prevention in minority populations: a systematic review and framework. The Diabetes Educator, 43, 54-77.

Larson, J. S. (1999). The conceptualization of health. Medical Care Research and Review, 56(2), $123-136$.

Layard, R., Clark, A., \& Senik, C. (2012). World Happiness REPORT 2012. In World Happiness Report 2013, edited by John Helliwell, Richard Layard, and Jeffrey Sachs. Retrieved from: https://s3.amazonaws. com/happiness-report/2012/World_Happiness_Report_2012.pdf [Online_Resource] (Accessed 01/09 /2019).

Mackenbach, J. P. (2012). The persistence of health inequalities in modern welfare states: the explanation of a paradox. Social Science and Medicine, 75, 761-769.

Marcolino, S. M., Oliveira, J. A. Q., D’Agostino, M., Rineiro, A. L., Alkmin, M. B. M., \& Novillo-Ortiz, D. (2018). The impact of mHealth interventions: Systematic review of systematic reviews. JMIR mHealth and uHealth, 6, e23. https://doi.org/10.2196/mhealth.8873.

Mason, S., Hussain-Gambles, M., Leese, B., Atkin, K., \& Brown, J. (2003). Representation of South Asian people in randomised clinical trials: analysis of trials' data. BMJ (Clinical Research ed.), 326, e1244. https://doi.org/10.1136/bmj.326.7401.1244.

Masood, Y., Bower, P., Waheed, M. W., Brown, G., \& Waheed, W. (2019). Synthesis of researcher reported strategies to recruit adults of ethnic minorities to clinical trials in the United Kingdom: a systematic review. Contemporary Clinical Trials, 78, 1-10. https://doi.org/10.1016/j.cct.2019.01.004.

Mendoza-Vasconez, A. S., Linke, S., Muñoz, M., Pekmezi, D., Ainsworth, C., Cano, M., Pkmezi, D., Ainsworth, C., Cano, M., Williams, V., Marcus, B. H., \& Larsen, B. A. (2016). Promoting physical activity among underserved populations. Current Sports Medicine Reports, 15, 290-297.

Montague, E., \& Perchonok, J. (2012). Health and wellness technology use by historically underserved health consumers: systematic review. Journal of Medical Internet Research, 14(3), e78. https://doi.org/10.2196 /jmir.2095.

Munn, Z., Tufanaru, C., \& Aromataris, E. (2014). JBI's systematic reviews: data extraction and synthesis. The American Journal of Nursing, 114, 49-54.

NHS Health Scotland (2016). Health inequalities - what are they and how do we reduce them? Retrieved from: http:/www.healthscotland.scot/media/1086/health-inequalities-what-are-they-how-do-we-reducethem-mar16.pdf [Online_Resource].

Phelan, J. C., Link, B. G., \& Tehranifar, P. (2010). Social conditions as fundamental causes of health inequalities: theory, evidence, and policy implications. Journal of Health and Social Behaviour, 51, S28-S40. https://doi.org/10.1177/0022146510383498.

Popay, J., Roberts, H., Sowden, A., Petticrew, M., Arai, L., Rodgers, M., Britten, N., Roen, K., \& Duffy, S., (2006). Guidance on the conduct of narrative synthesis in systematic reviews. A product from the ESRC methods programme. Version 1. ESRC. Retrieved: https://www.lancaster.ac. $\mathrm{uk} / \mathrm{shm} / \mathrm{research} / \mathrm{nssr} / \mathrm{research} /$ dissemination/publications/NS_Synthesis_Guidance_v1.pdf [Online_ Resource].

Popay, J., Whitehead, M., Carr-Hill, R., Dibben, C., Dixon, P., Halliday, E., Nazroo, J., Pearl, E., Povall, S., Srafford, M., Turner, J., \& Walthery, P. (2015). The impact on health inequalities of approaches to 
community engagement in the new deal for communities regeneration initiative: a mixed-methods evaluation. Public Health Research, 3(12), 167. https://doi.org/10.3310/phr03120.

Popkin, B. M., Duffey, K., \& Gordon-Larsen, P. (2005). Environmental influences on food choice, physical activity and energy balance. Physiology \& Behaviour, 86(5), 603-613.

Price Waterhouse Coopers (2013). Socio-economic impact of mHealth: an assessment report for the European Union. Retrieved from https:/www.gsma.com/iot/wp-content/uploads/2013/06/Socio-economic_impactof-mHealth_EU_14062013V2.pdf [Online_Resource].

Roosa, M., Liu, F., Torres, M., Gonzales, N., Knight, G., \& Saenz, D. (2008). Sampling and recruitment in studies of cultural influences on adjustment: a case study with Mexican Americans. Journal of Family Psychology, 22, 293-302.

*Ruggiano, N., Brown, E., Li, J., \& Scaccianoce, M. (2018). Rural caregivers and technology: what is the evidence? Research in Gerontological Nursing, 11, 216-224.

Schueller, S. M., Hunter, J. F., Figueroa, C. A., \& Aguilera, A. (2019). Use of digital mental health for marginalized and underserved populations. Current Treatment Options in Psychiatry, 6, 243-255.

Shea, B. J., Reeves, B. C., Wells, G., Thuku, M., Hamel, C., Moran, J., Moher, D., Tugwell, P., Welch, V., Kristjansson, R., \& Henry, D. A. (2017). AMSTAR 2: a critical appraisal tool for systematic reviews that include randomised or non-randomised studies of healthcare interventions, or both. BMJ (Clinical Research Ed.), 358, j4008. https://doi.org/10.1136/bmj.j4008.

*Sinclair, C. (2015). Effectiveness and user acceptance of online chronic disease management interventions in rural and remote setting: systematic review and narrative synthesis. Clinical Medicine Insights: Therapeutics, 7(7), 43-52.

Srinivasan, S., O’Fallon, L. R., \& Dearry, A. (2003). Creating healthy communities, healthy homes, healthy people: initiating a research agenda on the built environment and public health. American Journal of Public Health, 93, 1446-1450.

Stiglitz, J.E., Sen, A., Fitoussi, J.P. (2009). Report by the commission on the measurement of economic performance and social progress. Retrieved from https://ec.europa.eu/eurostat/documents/118025 /118123/Fitoussi+Commission+report [Online_Resource]. (Accessed 01/08/2019).

Waheed, W., Hughes-Morley, A., Woodham, A., Allen, G., \& Bower, P. (2015). Overcoming barriers to recruiting ethnic minorities to mental health research: a typology of recruitment strategies. $B M C$ Psychiatry, 15, 101-112.

Walsh, D. M. J., Moran, K., Cornelissen, V., Buys, R., Claes, J., Zampognaro, P., Melillo, F., Maglaveras, N., Chouvarda, I., Triantafullidis, A., Filos, D., \& Woods, C. B. (2019). The development and codesign of the PATHway intervention: a theory-driven eHealth platform for the self-management of cardiovascular disease. Translational Behavioural Medicine, 9(1), 76-98.

Weinert, C., \& Hill, W. G. (2005). Rural women with chronic illness: Computer use and skill acquisition. Womens Health Issues, 15(5), 230-236.

World Health Organization (2012a). Management of Patient Information: Based on the Findings of the Second Global Survey on eHealth, Global observatory for eHealth Series, volume 6. Retrieved from: https:/www.who.int/goe/publications/ehealth_series_vol6/en/ [Online Resource],

World Health Organization (2012b). Health Impact Assessment (HIA) - Glossary of terms used. Retrieved from: http://www.who.int/hia/about/glos/en/index1.html [Online_Resource].

Yardley, L., Morrison, L., Bradbury, K., \& Muller, I. (2015). The person-based approach to intervention development: application to digital health-related behavior change interventions. Journal of Medical Internet Research, 17(1), e30. https://doi.org/10.2196/jmir.4055.

Publisher's Note Springer Nature remains neutral with regard to jurisdictional claims in published maps and institutional affiliations.

\section{Affiliations}

\section{Maria Armaou $^{1} \cdot$ Evangelia Araviaki $^{2} \cdot$ Laura Musikanski $^{3}$}

1 University of Nottingham, Nottingham, UK

2 Coventry, UK

3 Happiness Alliance, Seattle, WA, USA 